\begin{tabular}{|c|l|}
\hline Title & $\begin{array}{l}\text { Superconductor-based Light Emitting Diode: Demonstration of Role of Cooper Pairs in Radiative Recombination } \\
\text { Processes }\end{array}$ \\
\hline Author(s) & Hay ashi, Y ujiro; Tanaka, Kazunori; A kazaki, Tatsushi; Jo, Masafumi; Kumano, Hidekazu; Suemune, Ikuo \\
\hline Citation & $\begin{array}{l}\text { A pplied Physics Express, 1, 011701 } \\
\text { https://doi.org/10.1143/APEX.1.011701 }\end{array}$ \\
\hline Issue Date & 2008 \\
\hline Doc URL & http://hdl.handle.net/2115/33908 \\
\hline Type & article (author version) \\
\hline File Information & 280-A PEX 08 text.pdf \\
\hline
\end{tabular}

Instructions for use 


\title{
Superconductor-based Light Emitting Diode: Demonstration of Role of Cooper Pairs in Radiative Recombination Processes
}

\author{
Yujiro Hayashi $^{1 *}$, Kazunori Tanaka ${ }^{2,3}$, Tatsushi Akazaki ${ }^{4,3}$, Masafumi Jo ${ }^{1}$, Hidekazu Kumano ${ }^{1,3}$ \\ and Ikuo Suemune $e^{1,3}$ \\ ${ }^{1}$ Research Institute for Electronic Science, Hokkaido University \\ ${ }^{2}$ Central Laboratory, Hamamatsu Photonics \\ ${ }^{3}$ CREST, Japan Science and Technology Corporation \\ ${ }^{4}$ NTT Basic Research Laboratory
}

\begin{abstract}
A light emitting diode with superconducting $\mathrm{Nb}$ electrodes was fabricated to investigate the contribution of Cooper pairs to radiative recombination in a semiconductor. Electroluminescence observed from the active layer in which electron Cooper pairs and normal holes are injected was drastically enhanced at the temperature lower than the superconducting transition temperature of the $\mathrm{Nb}$ electrodes. This is the first experimental evidence that Cooper pairs enhance radiative recombinations by the superradiance effect.
\end{abstract}

Superconducting quantum bits (qubits) are one of the most promising candidates for quantum information processing because coherent qubit operations are possible with conventional microwave techniques based on electrical circuits prepared with conventional lithography techniques. The entanglement and quantum logic gates of superconducting qubits have been successfully demonstrated. ${ }^{1,2)}$ On the other hand, for the transportation of qubits, photons are most suitable for quantum links based on optical-fiber communication networks. Photon qubits and their applications to quantum cryptography have been actively studied. ${ }^{3)}$ However the two research fields are isolated at present, and new technique connecting superconducting qubits with photon qubits will be indispensable for constructing quantum information networks.

Electrically driven single photon sources using exciton emission in semiconductor quantum dots (QDs) have been successfully demonstrated. ${ }^{4)}$ Entangled photon pair generation from QDs is also expected by using biexciton-exciton cascade emission. ${ }^{5)}$ However, it has been difficult in most of the cases to achieve the quantum entanglement of biexciton and exciton photon pairs mainly due to lift-off of spin degeneracy of intermediate exciton states caused by crystallographic anisotropy of semiconductor surfaces and electron-hole exchange interactions. ${ }^{6)}$ Time intervals and different time extensions of biexciton and exciton photon wave packets due to the difference of their radiative recombination lifetimes will prevent the application to more sophisticated quantum information processing.

\footnotetext{
*E-mail address: yhayashi@es.hokudai.ac.jp
} 
Recently, we have proposed a new scheme to realize solid-state entangled photon pair sources using superconductors, ${ }^{7)}$ where Cooper pairs recombine simultaneously bypassing intermediate exciton states in semiconductor QDs. This was named as superconductor-based quantum-dot light emitting diodes (SQ-LED), and Cooper pair injection into semiconductors was demonstrated using $\mathrm{Nb} / \mathrm{n}-\mathrm{InGaAs} / \mathrm{Nb}$ vertical Josephson junction, which shows that electron Cooper-pairs tunnel through 120-nm-thick InGaAs layers with In concentrations of $53 \sim 70 \%$. This proposal was based on the superradiance effect on electron and hole Cooperpair recombinations theoretically predicted by E. Hanamura. ${ }^{8)}$ However this prediction has not been confirmed experimentally.

In this paper, as the first step to realize the scheme of the SQ-LED, the contribution of Cooper pairs to radiative recombination in a semiconductor was examined experimentally by using a LED with superconductor $\mathrm{Nb}$ electrodes, in which electron Cooper pairs will be injected into a $\mathrm{n}$-InGaAs active layer from the superconducting $\mathrm{Nb}$ electrodes by the proximity effect. It is noted that the recombination process dealt with in this paper is the one between electron Cooper pairs and normal holes, since the recombination between electron and hole Cooper pairs assumed in ref. 8 is technologically difficult to realize with direct-bandgap semiconductors.

The superconductor-based LED as illustrated in Fig. 1 was prepared on a p-type (100) InP substrate. The heterostructure grown by metal-organic vapor-phase epitaxy (MOVPE) consists of a 500-nm-thick $\mathrm{p}^{+}-\mathrm{InP}$ buffer layer, (i) a 10 -nm-thick $\mathrm{n}^{+}-\mathrm{In}_{0.6} \mathrm{Ga}_{0.4}$ As active layer, (ii) a 20-nm-thick $\mathrm{n}^{++}-\mathrm{In}_{0.53} \mathrm{Ga}_{0.47}$ As barrier layer lattice-matched to $\mathrm{InP}$, and (iii) a 10nm-thick $\mathrm{n}^{++}-\mathrm{In}_{0.7} \mathrm{Ga}_{0.3}$ As ohmic contact layer. To increase the coherence length of electron Cooper pairs penetrated from $\mathrm{Nb}$ electrode deposited on the InGaAs contact layer into the n-InGaAs layers by the proximity effect, the InGaAs contact and barrier layers were highly doped to $5 \times 10^{18} \mathrm{~cm}^{-3}$. After the removal of surface oxide of the ohmic contact layer with Ar-ion sputtering in a $\mathrm{Nb}$ evaporation chamber, the $\mathrm{Nb}$ cathode was deposited with electron beam deposition. The area of the $\mathrm{Nb}$ cathode in contact to the n-InGaAs surface was limited to $50 \times 50 \mu \mathrm{m}^{2}$ by employing SiN mask opening and the lift-off technique of $\mathrm{Nb}$ electrodes. After the removal of photoresist for selective etching of the InGaAs layers in the area out

\begin{tabular}{|c|c|c|}
\hline $80 \mathrm{~nm}$ & $\stackrel{110 \mathrm{~nm}}{\rightarrow}$ & $\mathrm{Nb}$ cathode \\
\hline $10 \mathrm{~nm}$ & $\mathrm{n}^{+}-\mathrm{In}_{07} \mathrm{Ga}_{03} \mathrm{As}$ & (iii) \\
\hline $20 \mathrm{~nm}$ & $\mathrm{n}^{+}-\mathrm{In}_{0.53} \mathrm{Ga}_{0.47} \mathrm{As}$ & (ii) \\
\hline $10 \mathrm{~nm}$ & $\mathrm{n}^{+}-\mathrm{In}_{06} \mathrm{Ga}_{04} \mathrm{As}$ & (i) \\
\hline $500 \mathrm{~nm}$ & $\mathrm{p}^{+}-\operatorname{In} \mathrm{P}$ & \\
\hline & p - InP substrate & \\
\hline & Au anode & \\
\hline
\end{tabular}

Fig. 1. Schematic structure of the superconductor-based LED. 


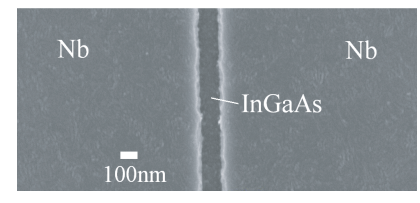

Fig. 2. SEM image of Nb cathode nano-slit.

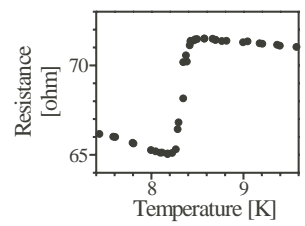

Fig. 3. Nb cathode resistance vs. temperature. Superconducting transition is seen in the Nb cathode at $T_{\mathrm{C}}=8.3 \sim 8.4 \mathrm{~K}$

of the $\mathrm{Nb}$ cathode, $\mathrm{Au} / \mathrm{Cr}$ contact pads were formed to have electrical contacts to the $\mathrm{Nb}$ cathodes by photoresist patterning and lift-off. Au anode was deposited to the back surface of the InP substrate. To observe electroluminescence (EL) from the n-InGaAs active layer, a slit on the $\mathrm{Nb}$ cathode was fabricated by electron-beam resist patterning and reactive ion etching. Although dry etching of high melting point metals such as $\mathrm{Nb}$ is generally difficult, the slit with the width of $\sim 110 \mathrm{~nm}$ was successfully formed as shown by the scanning electron microscope (SEM) image in Fig. 2. Two device chips with and without the $\mathrm{Nb}$ slit were prepared for the measurement of EL and superconducting critical temperature $T_{\mathrm{C}}$ of the $\mathrm{Nb}$ electrode, respectively, by cleaving the processed wafer into the area of $0.5 \times 0.5 \mathrm{~mm}^{2}$.

Measurements were conducted using a ${ }^{3} \mathrm{He}$ refrigerator cryostat equipped with optical fibers. From the measurement of the $\mathrm{Nb}$ cathode resistance as a function of temperature $T$ as shown in Fig. 3, $T_{\mathrm{C}}$ was estimated to be $8.3 \sim 8.4 \mathrm{~K}$. The residual resistance and its slight temperature dependence in the temperature range below $T_{\mathrm{C}}$ may be due to oxidation of the $\mathrm{Nb}$ surface and additional influence of remaining thin resist layer due to the photoresist processes on the $\mathrm{Nb}$ surface. The EL spectra were measured through an optical fiber with the core diameter of $200 \mu \mathrm{m}$ located $\sim 0.5 \mathrm{~mm}$ above the sample and by an infrared grating spectrometer equipped with an InGaAs photodiode array detector.

The heterostructure band diagram toward the depth direction in Fig. 1 for the given doping profile under a forward bias was calculated assuming quasi-Fermi level separation of $\mathrm{eV}=E_{\mathrm{Fn}}-E_{\mathrm{Fp}}=1.1 \mathrm{eV}$ at the boundary between the p-type InP and n-type InGaAs active layer as shown in Fig. 4. Because of the heavy n-type doping in the InGaAs layers, the Fermi energy in the InGaAs heterostructure is positioned very close to the conduction band. This will reduce the effective potential barriers for electron Cooper pairs to penetrate from the $\mathrm{Nb}$ superconducting electrode into the InGaAs layers by the proximity effect at $T<T_{\mathrm{C}}$. When 


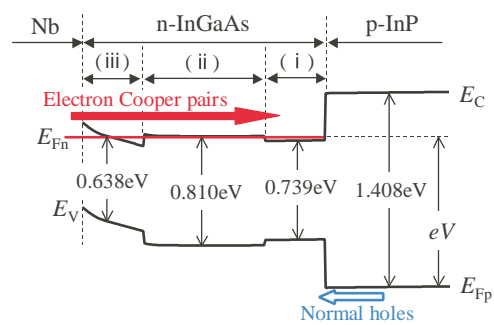

Fig. 4. Calculated band structure of the LED under a forward bias
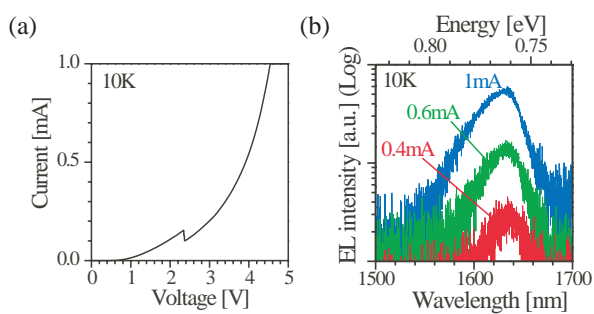

Fig. 5. Typical $I-V$ curve (a) and EL (b) of the LED at $T>T_{\mathrm{C}}$.

the diode is forward-biased, the electron Cooper pairs are expected to penetrate into the InGaAs active layer following our previous measurement ${ }^{7)}$ and will encounter normal holes injected from the p-InP. The energy difference between the electron and hole ground states in the $\mathrm{In}_{0.6} \mathrm{Ga}_{0.4} \mathrm{As}$ quantum well (QW) active layer was estimated to be $0.762 \mathrm{eV}$ by calculating the one-dimensional time-independent Schrödinger equation for an asymmetric finite square well potential using the band gap energy $E_{\mathrm{g}}$ as listed in Fig. 4 and conduction band offset of $0.65 \Delta E_{\mathrm{g}}$ at $10 \mathrm{~K}$.

Figures 5(a) and 5(b) show a forward-biased current-voltage $(I-V)$ curve and an EL spectrum measured through the Nb cathode nano-slit at $10 \mathrm{~K}$ of which temperature is higher than $T_{\mathrm{C}}$ with the diode current to $1 \mathrm{~mA}$, respectively. The small negative differential resistance (NDR) shown in Fig. 5(a) has been observed in some of the samples with similar structures, but it has not been observed with the InGaAs heterostructures with less hetero-barriers. The origin of the NDR will most probably be related to instability related to charge accumulation at hetero-interfaces and their field screening effect. ${ }^{9)}$ This NDR will not play a major role in the present issue related to superconductivity. The observed EL peak of $\sim 0.760 \mathrm{eV}$ was almost invariant against the injection current and was in reasonable agreement with the calculated transition energy of the QW active layer. Although the InGaAs detector used in this study showed lower sensitivity below the energy of $0.756 \mathrm{eV}$, the injection current dependence of the EL intensity was not influenced by this factor. In Fig. 5(b), the higher energy tail slightly increased with the increase of the injection current. This will be due to the superposition 


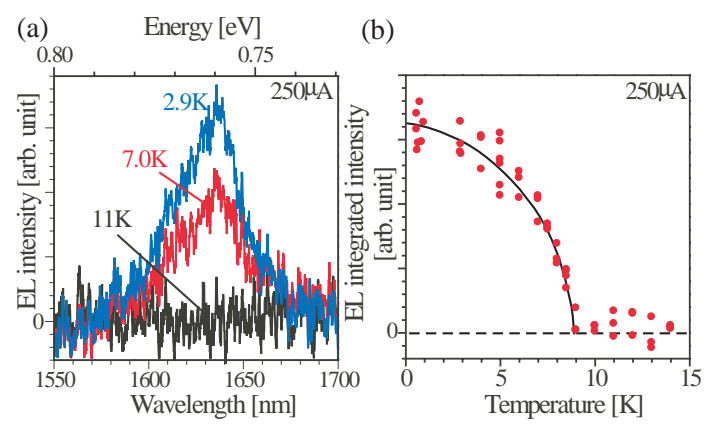

Fig. 6. Temperature dependence of EL spectra (a) and EL integrated intensity (b) where the solid line is guide to the eye.

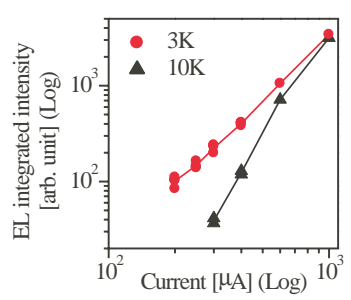

Fig. 7. Temperature dependence of EL integrated intensity vs. current.

of the luminescence from the barrier layer. In addition to this EL measurements, microphotoluminescence measurements were performed, in which the luminescence spectra with high photo-excitation was expanded to the higher energy and additional peak around $0.80 \mathrm{eV}$ corresponding to the barrier layer was observed. In this paper, the main discussion will be limited to the lower current region so that the emission from the QW active layer dominates.

To examine the role of the electron Cooper pairs penetrating from the superconducting $\mathrm{Nb}$ electrode to the InGaAs active layer, the temperature dependence of EL was examined around $T_{\mathrm{C}}$ under the constant current of $250 \mu \mathrm{A}$. The EL spectra remained noise level at the temperature higher than $\sim 9 \mathrm{~K}$ as exemplified with the result at $11 \mathrm{~K}$ in Fig. 6 (a). But the EL from the active layer was enhanced and clearly observed at the lower temperature as exemplified with the EL spectra at $7.0 \mathrm{~K}$ and $2.9 \mathrm{~K}$ in Fig. $6(\mathrm{a})$. The EL integrated intensities are plotted as a function of $T$ in Fig. 6(b). The dramatic increase of the EL intensity at the temperature lower than $\sim 9 \mathrm{~K}$ will be clear, while the EL intensity is under measurement limit at the higher temperature. The apparent critical temperature of $\sim 9 \mathrm{~K}$ for the EL intensity enhancement finely agrees with the superconductivity of $T_{\mathrm{C}} \sim 8.4 \mathrm{~K}$. The abrupt increase of the EL intensity for the lower temperature just below $T_{\mathrm{C}}$ and the gradual saturation of the EL intensity approaching $0 \mathrm{~K}$ will be accounted for by the temperature dependence of the superconducting energy gap, i.e., abrupt increase at $T \leq T_{\mathrm{C}}$ and saturation at $0 \leq T \ll T_{\mathrm{C}}$. ${ }^{10}$ )

The enhancement of the radiative recombinations by the penetration of electron Cooper 
pairs in the InGaAs active layer will be controlled by the temperature dependence of the coherence length at $T<T_{\mathrm{C}}$. The observation of negligible EL at $11 \mathrm{~K}$ shown in Fig. 6(a) suggests that non-radiative recombinations are dominant at $T>T_{\mathrm{C}}$ under the bias condition of $250 \mu \mathrm{A}$. The bias dependence of the EL intensity was compared in Fig. 7 at the two temperatures of $3 \mathrm{~K}$ and $10 \mathrm{~K}$ which are below and above $T_{\mathrm{C}}$, respectively. It is noted that the EL spectra measured under the constant injection current with different temperature were almost completely overlapped with the normalization of their intensity in the temperature range below $13 \mathrm{~K}$. This confirms that the temperature dependence of the EL intensity shown in Figs. 6(b) and 7 is not due to the spectral change but due to the Cooper-pair effect injected to the InGaAs active layer. The improvement of the EL intensity at $3 \mathrm{~K}$ becomes more enhanced at the lower injection current. Generally mid-gap deep levels are more active under the lower injection current and non-radiative recombinations will dominate. Therefore the above results shown in Fig. 7 indicate that the enhancement of the radiative recombination rate due to the Cooper pairs comes out more evidently under the situation with otherwise lower internal quantum efficiency.

In conclusion, LEDs with Nb superconducting electrodes were fabricated. Drastic enhancement of the EL intensity was observed at the temperature lower than the superconducting critical temperature. This is the first experimental evidence that electron Cooper pairs enhance radiative recombinations in semiconductors by the superradiance effect.

The authors are grateful to Dr.M.Endo of Hokkaido Univ. and Hokkaido Innovation through NanoTechnology Support (HINTS) for nanofabrication, Mr.S.Furuta of Hamamatsu Photonics and Dr.T.Maruyama of NTT BRL for sample preparations, Prof.H.Takayanagi and Dr.R.Inoue of Tokyo Univ. of Science for related electrical measurements, and Dr.Y.Asano, Dr.Y.Idutsu of Hokkaido Univ. and Dr.K.Uesugi of Muroran Institute of Technology for fruitful discussions. 


\section{References}

1) M. Steffen, M. Ansmann, R. C. Bialczak, N. Katz, E. Lucero, R. McDermott, M. Neeley, E. M. Weig, A. N. Cleland, and J. M. Martinist: Science 313 (2006) 1423.

2) J. H. Plantenberg, P. C. de Groot, C. J. P. M. Harmans, and J. E. Mooij: Nature 447 (2007) 836.

3) for example, N. Gisin, G. Ribordy, W. Tittel, and H. Zbinden: Rev. Mod. Phys. 74 (2002) 145.

4) Z. Yuan, B. E. Kardynal, R. M. Stevenson, A. J. Shields, C. J. Lobo, K. Cooper, N. S. Beattie, D. A. Ritchie, and M. Pepper: Science 295 (2002) 102.

5) R. M. Stevenson, R. J. Young, P. Atkinson, K. Cooper, D. A. Ritchie, and A. J. Shields: Nature 439 (2006) 179.

6) V. D. Kulakovskii, G. Bacher, R. Weigand, T. Kümmell, A. Forchel, E. Borovitskaya, K. Leonardi, and D. Hommel: Phys. Rev. Lett. 82 (1999) 1780.

7) I. Suemune, T. Akazaki, K. Tanaka, M. Jo, K. Uesugi, M. Endo, H. Kumano, E. Hanamura, H. Takayanagi, M. Yamanishi, and H. Kan: Jpn. J. Appl. Phys. 45 (2006) 9264.

8) E. Hanamura: Phs. Stat. Solid. (b) 234 (2002) 166.

9) K. Uesugi, M. Kurimoto, I. Suemune, M. Yamamoto, T. Uemura, H. Machida, and N. Shimoyama: Physica E 21 (2004) 727.

10) for example, M. Tinkham: Introduction to Superconductivity (McGraw-Hill Book, New York, 1975). 